PROCEEDINGS OF THE

AMERICAN MATHEMATICAL SOCIETY

Volume 139, Number 4, April 2011, Pages 1201-1213

S 0002-9939(2010)10623-8

Article electronically published on September 29, 2010

\title{
ŁOJASIEWICZ EXPONENT NEAR THE FIBRE OF A MAPPING
}

\author{
TOMASZ RODAK AND STANISŁAW SPODZIEJA
}

(Communicated by Ted Chinburg)

\begin{abstract}
Let $g: X \rightarrow \mathbb{R}^{k}$ and $f: X \rightarrow \mathbb{R}^{m}$, where $X \subset \mathbb{R}^{n}$, be continuous semi-algebraic mappings, and $\lambda \in \mathbb{R}^{m}$. We describe the optimal exponent $\theta=: \mathcal{L}_{\infty, f \rightarrow \lambda}(g)$ for which the Eojasiewicz inequality $|g(x)| \geqslant C|x|^{\theta}$ holds with $C>0$ as $|x| \rightarrow \infty$ and $f(x) \rightarrow \lambda$. We prove that there exists a semi-algebraic stratification $\mathbb{R}^{m}=S_{1} \cup \cdots \cup S_{j}$ such that the function $\lambda \mapsto \mathcal{L}_{\infty, f \rightarrow \lambda}(g)$ is constant on each stratum $S_{i}$. We apply this result to describe the set of generalized critical values of $f$.
\end{abstract}

\section{INTRODUCTION}

Let $M, N, L$ be finite-dimensional real vector spaces, $X \subset M$ be a closed semialgebraic set, $g: X \rightarrow N$ and $f: X \rightarrow L$ be continuous semi-algebraic mappings (see [1), and let $\lambda \in L$. The aim of this article is to describe the Eojasiewicz exponent at infinity of $g$ near the fibre $f^{-1}(\lambda)$, i.e. the supremum of the exponents $\theta$ for which the Eojasiewicz inequality

$$
|g(x)| \geqslant C|x|^{\theta} \quad \text { as } \quad x \in X, \quad|x| \rightarrow \infty \quad \text { and } \quad f(x) \rightarrow \lambda
$$

holds with $C>0$ (cf. [12, [18), where $|\cdot|$ is a norm. We denote this exponent by $\mathcal{L}_{\infty, f \rightarrow \lambda}(g)$ (see Section 1 for details).

We prove that $\mathcal{L}_{\infty, f \rightarrow \lambda}(g) \in \mathbb{Q} \cup\{-\infty,+\infty\}$ for $\lambda \in L$ and that there exists a semi-algebraic stratification $L=S_{1} \cup \cdots \cup S_{j}$ such that the function $\lambda \mapsto \mathcal{L}_{\infty, f \rightarrow \lambda}(g)$ is constant on each stratum $S_{i}$ (Theorem 1.2). If $g$ and $f$ are complex regular mappings, the stratification is complex algebraic (Corollary 1.6). Note that if $\theta=$ $\mathcal{L}_{\infty, f \rightarrow \lambda}(g) \in \mathbb{Q}$, then $(\mathbb{E})$ holds (Corollary 3.7). The key points in the proofs are Lipschitz stratifications ([13, [14], 20]) and properties of the set of points at which a mapping is not proper ([8]; see also Section 2 2).

If $f: M \rightarrow L$ is a semi-algebraic mapping of class $\mathscr{C}^{1}$, we define the Eojasiewicz exponent of $d f$ near the fibre $f^{-1}(\lambda)$ by

$$
\mathcal{L}_{\infty, \lambda}(f)=\mathcal{L}_{\infty, f \rightarrow \lambda}(\nu(d f)),
$$

where $\nu$ is a function introduced by Rabier 17] (see Section 11). This notion was introduced by $\mathrm{Ha}$ [7] in the case of complex polynomial functions in two variables (see also [3, [5]).

Received by the editors May 19, 2009 and, in revised form, April 19, 2010.

2010 Mathematics Subject Classification. Primary 14R25; Secondary 58K55, 58K05.

Key words and phrases. Łojasiewicz exponent at infinity, generalized critical values, stratification.

This research was partially supported by the program POLONIUM.

(C)2010 American Mathematical Society 
Let us recall that the exponent $\mathcal{L}_{\infty, \lambda}(f)$ is strongly related to the set of bifurcation points of $f$. Namely, one can define the set of generalized critical values of $f$ by

$$
K_{\infty}(f)=\left\{\lambda \in L: \mathcal{L}_{\infty, \lambda}(f)<-1\right\} .
$$

It is a closed and semi-algebraic set. By Theorem 1.2, the mapping $L \ni \lambda \mapsto$ $\mathcal{L}_{\infty, \lambda}(f)$ has a finite number of values (Corollary 1.5); hence there exists $\alpha>0$ such that

$$
K_{\infty}(f)=\left\{\lambda \in L: \mathcal{L}_{\infty, \lambda}(f)<-1-\alpha\right\} .
$$

If $f$ is of class $\mathscr{C}^{2}$, then for any $\lambda \in L \backslash K_{\infty}(f)$ there exist a neighbourhood $U \subset L$ of $\lambda$ and a compact set $\Delta \subset M$ such that $f: f^{-1}(U) \backslash \Delta \rightarrow U$ is a trivial bundle (see [16], 17], 11]; see also [23], 21], 22], [7, 15] for polynomials and polynomial mappings). The smallest set $B \subset L$ such that $L \backslash B$ has the above property is called the bifurcation set at infinity of $f$ and is denoted by $B_{\infty}(f)$. Note that for a complex polynomial $f$ in two variables, $B_{\infty}(f)=K_{\infty}(f)$ (see [7], [15]).

Chądzyński and Krasiński ([3], Corollary 4.7) proved that for a complex polynomial $f$ in two variables with $\operatorname{deg} f>0$ there exists $c_{f} \in \mathbb{Q}$ with $c_{f} \geqslant 0$ such that

$$
\mathcal{L}_{\infty, \lambda}(f)=c_{f} \text { for } \lambda \notin K_{\infty}(f) \quad \text { and } \quad \mathcal{L}_{\infty, \lambda}(f)<-1 \text { for } \lambda \in K_{\infty}(f) .
$$

They also asked whether $\lambda \mapsto \mathcal{L}_{\infty, \lambda}(f)$ behaves similarly in the general case. Note that in the multi-dimensional case we cannot require $c_{f} \geqslant 0$. Indeed, for the polynomial $f\left(z_{1}, z_{2}, z_{3}\right)=\left(z_{1} z_{2}-1\right) z_{2} z_{3}$ ([17, Remark 9.1) we have $c_{f}=-1$ (see [3], Proposition 6.4).

As a corollary from Theorem 1.2 we give a partial answer to the above-mentioned question. Namely, for a nonconstant polynomial $f: \mathbb{C}^{n} \rightarrow \mathbb{C}$ there exist a finite set $S \subset \mathbb{C}$ with $K_{\infty}(f) \subset S$ and $c_{f} \geqslant-1$ such that $\mathcal{L}_{\infty, \lambda}(f)=c_{f}$ for $\lambda \in \mathbb{C} \backslash S$ and $\mathcal{L}_{\infty, \lambda}(f)<c_{f}$ for $\lambda \in S$ (Corollary 1.7). It is not clear to the authors whether $S=K_{\infty}(f)$ in Corollary 1.7 .

Section 2 has an auxiliary character and contains some results on semi-algebraic mappings, Łojasiewicz exponent and stratifications. In Sections 3 and 4 we prove Theorem 1.2 and Corollary [1.6, respectively.

\section{LOJASIEWICZ EXPONENT NEAR THE FIBRE OF A MAPPING}

Let $M, N, L$ be finite-dimensional real vector spaces, $X \subset M$ be a closed set, let $g: X \rightarrow N$ and $f: X \rightarrow L$, and let $\lambda \in L$.

Definition 1.1. By the Eojasiewicz exponent at infinity of $g$ near the fibre $f^{-1}(\lambda)$ we mean

$$
\mathcal{L}_{\infty, f \rightarrow \lambda}(g):=\sup \left\{\mathcal{L}_{\infty}\left(g \mid f^{-1}(U)\right): U \subset L \text { is a neighbourhood of } \lambda\right\},
$$

where

$$
\mathcal{L}_{\infty}(g \mid S):=\sup \left\{\theta \in \mathbb{R}: \exists_{C, R>0} \forall_{x \in S}\left(x \geqslant R \Rightarrow|g(x)| \geqslant C|x|^{\theta}\right)\right\}
$$

is the Lojasiewicz exponent at infinity of $g$ on a set $S \subset X$.

Our main result is

Theorem 1.2. Let $g: X \rightarrow N$ and $f: X \rightarrow L$ be continuous semi-algebraic mappings.

(i) For any $\lambda \in L, \mathcal{L}_{\infty, f \rightarrow \lambda}(g) \in \mathbb{Q} \cup\{-\infty,+\infty\}$. 
(ii) The function

$$
\vartheta_{g / f}: L \ni \lambda \mapsto \mathcal{L}_{\infty, f \rightarrow \lambda}(g)
$$

is upper semi-continuous, and there exists a semi-algebraic stratification

$$
L=S_{1} \cup \cdots \cup S_{j}
$$

such that $\vartheta_{g / f}$ is constant on each stratum $S_{i}, i=1, \ldots, j$.

The proof of Theorem 1.2 is given in Section 3. Theorem 1.2(ii) was proved in [18] for complex polynomials, under the assumption (i).

Now let $f: M \rightarrow L$ be a semi-algebraic mapping of class $\mathscr{C}^{1}$ and let $d f$ be the differential of $f$. Let

$$
\nu(d f): M \ni x \mapsto \nu(d f(x)) \in \mathbb{R},
$$

be the Rabier function, i.e. for $A=d f(x): M \rightarrow L$,

$$
\nu(A)=\inf _{\|\phi\|=1}\left\|A^{*}(\phi)\right\|,
$$

where $A^{*}: L^{*} \rightarrow M^{*}$ is the adjoint operator and $\phi \in L^{*}$. For a semi-algebraic function $f: M \rightarrow \mathbb{R}$ (or a complex polynomial) we have $\nu(d f)=|\nabla f|$, where $\nabla f$ is the gradient of $f$.

Definition 1.3. The Eojasiewicz exponent of $d f$ near a fibre $f^{-1}(\lambda)$ is defned to be $\mathcal{L}_{\infty, \lambda}(f)=\mathcal{L}_{\infty, f \rightarrow \lambda}(\nu(d f))$.

Remark 1.4. Let $f: \mathbb{R}^{n} \rightarrow \mathbb{R}^{m}$ be a semi-algebraic mapping of class $\mathscr{C}^{1}$ and let $\kappa(d f): \mathbb{R}^{n} \ni x \mapsto \kappa(d f(x)) \in \mathbb{R}$ be the Kuo function [10]; i.e., for $A=d f(x)=$ $\left(A_{1}, \ldots, A_{m}\right): \mathbb{R}^{n} \rightarrow \mathbb{R}^{m}$,

$$
\kappa(A)=\min _{1 \leqslant i \leqslant m} \operatorname{dist}\left(\nabla A_{i},\left\langle\nabla A_{j}\right\rangle_{j \neq i}\right),
$$

where $\left\langle a_{j}\right\rangle_{j \neq i}$ is the vector space generated by the vectors $\left(a_{j}\right)_{j \neq i}$. As $\nu(A) \leqslant$ $\kappa(A) \leqslant \sqrt{m} \nu(A)([11$, Proposition 2.6), for any $\lambda \in L$ we have

$$
\mathcal{L}_{\infty, \lambda}(f)=\mathcal{L}_{\infty, f \rightarrow \lambda}(\kappa(d f)) .
$$

An analogous result holds for the Gaffney function [4] (cf. 9], Proposition 2.3).

The function $\nu(d f)$ is continuous and semi-algebraic (11], Proposition 2.4), so Theorem 1.2 implies:

Corollary 1.5. Let $f: M \rightarrow L$ be a semi-algebraic mapping of class $\mathscr{C}^{1}$. Then $\mathcal{L}_{\infty, \lambda}(f) \in \mathbb{Q} \cup\{-\infty,+\infty\}$ for any $\lambda \in L$, and the function $L \ni \lambda \mapsto \mathcal{L}_{\infty, \lambda}(f)$ is upper semi-continuous and has a finite number of values. In particular, there exists $\alpha>0$ such that

$$
K_{\infty}(f)=\left\{\lambda \in L: \mathcal{L}_{\infty, \lambda}(f)<-1-\alpha\right\} .
$$

In the case of complex regular mappings, from Theorem 1.2 we obtain:

Corollary 1.6. Let $X \subset \mathbb{C}^{n}$ be a complex algebraic set, and let $g: X \rightarrow \mathbb{C}^{m}$ and $f: X \rightarrow \mathbb{C}^{k}$ be complex regular mappings. Then there exists a complex algebraic stratification $\mathbb{C}^{k}=S_{1} \cup \cdots \cup S_{j}$ such that the function

$$
\vartheta_{g / f}: \mathbb{C}^{k} \ni \lambda \mapsto \mathcal{L}_{\infty, f \rightarrow \lambda}(g) \in \mathbb{Q} \cup\{-\infty,+\infty\}
$$

is constant on each stratum $S_{i}, i=1, \ldots, j$. Moreover, $\vartheta_{g / f}$ is upper semicontinuous. 
The proof of the above corollary will be given in Section 4. The crucial fact in the proof is that $\vartheta_{g / f}\left(\mathbb{C}^{n}\right)=\vartheta_{g / f}\left(\mathbb{R}^{2 n}\right)$ and this set is finite (Theorem 1.2).

For a complex polynomial $f: \mathbb{C}^{n} \rightarrow \mathbb{C}$ the set $K_{\infty}(f)$ is finite (Proposition 2.4 see also [11, Theorem 3.1); hence Corollary 1.6 gives:

Corollary 1.7. Let $f: \mathbb{C}^{n} \rightarrow \mathbb{C}$ be a polynomial function with $\operatorname{deg} f>0$. Then there exist a finite set $S \subset \mathbb{C}$ with $K_{\infty}(f) \subset S$ and a constant $c_{f} \in \mathbb{Q}$ with $c_{f} \geqslant-1$ such that $\mathcal{L}_{\infty, \lambda}(f)=c_{f}$ for $\lambda \in \mathbb{C} \backslash S$, and $\mathcal{L}_{\infty, \lambda}(f)<c_{f}$ for $\lambda \in S$.

\section{Auxiliary Results}

In what follows, $L, M, N$ are finite-dimensional real vector spaces. We will use the Euclidean norm $|\cdot|$ in $M$ (or in $N, L)$. For $A \subset M$, let $\varrho(\cdot, A)$ denote the distance function to $A$, i.e. $\varrho(x, A)=\inf _{y \in A}|x-y|$ if $A \neq \emptyset$, and $\varrho(x, \emptyset)=1$.

2.1. Semi-algebraic mappings. A subset of $M$ is called semi-algebraic if it is defined by a finite alternative of finite systems of inequalities $P>0$ or $P \geqslant 0$, where $P$ are polynomials on $M$ (see [1, 2]). A mapping $f: X \rightarrow N$, where $X \subset M$, is called semi-algebraic if the graph $\Gamma(f)$ of $f$ is a semi-algebraic set. For instance, the distance to a semi-algebraic set is a semi-algebraic function (cf. 2]):

Proposition 2.1. Let $V \subset M$ be a semi-algebraic set. Then the function $\varrho_{V}$ : $M \ni x \mapsto \varrho(x, V) \in \mathbb{R}$ is continuous and semi-algebraic.

Let $X \subset M$ and let $f: X \rightarrow N$ be any mapping. We say (cf. [8]) that $f$ is proper at a point $y \in N$ if there exists an open neighbourhood $U$ of $y$ such that $f: f^{-1}(U) \rightarrow U$ is a proper map. The set of points at which $f$ is not proper is denoted by $\mathfrak{S}_{f}$. It is obvious that the set $\mathfrak{S}_{f}$ is closed. It is known that for a complex algebraic set $X \subset \mathbb{C}^{n}$ and a complex regular mapping $f: X \rightarrow \mathbb{C}^{m}$, the set $\mathfrak{S}_{f}$ is complex algebraic.

Proposition 2.2. Let $X$ be a closed semi-algebraic set. If the mapping $f: X \rightarrow N$ is semi-algebraic, then the set $\mathfrak{S}_{f}$ is also semi-algebraic.

Proof. Since $X$ is a closed set, we have

$$
\mathfrak{S}_{f}=\left\{y \in N: \forall_{A, \varepsilon>0} \exists_{x \in X}|x|>A \wedge|f(x)-y|<\varepsilon\right\} .
$$

Then, by the Tarski-Seidenberg Theorem, we obtain the assertion.

Let $f: X \rightarrow N$ with $X \subset M$. The degree of $f$ is defined by

$$
\operatorname{deg} f=\inf \left\{\theta \in \mathbb{R}: \exists_{C, R>0} \forall_{x \in X}\left(|x| \geqslant R \Rightarrow|f(x)| \leqslant C|x|^{\theta}\right)\right\} .
$$

Set $\operatorname{supp} f=\{x \in X: f(x) \neq 0\}$.

A curve $\varphi:[r,+\infty) \rightarrow M$ is called meromorphic at $+\infty$ if $\varphi$ is the sum of a Laurent series of the form

$$
\varphi(t)=a_{p} t^{p}+a_{p-1} t^{p-1}+\cdots, \quad a_{i} \in M, \quad p \in \mathbb{Z} .
$$

In the case of a polynomial function and the Laurent series at infinity, the above degree is the usual degree; that is, $\operatorname{deg} \varphi=p$ if $a_{p} \neq 0$, and $\operatorname{deg} \varphi=-\infty$ if $\varphi \equiv 0$.

Proposition 2.3. Let $X$ be a closed semi-algebraic set and let $f: X \rightarrow N$ be a semi-algebraic mapping. Then:

(i) $\operatorname{deg} f \in \mathbb{Q} \cup\{-\infty\}$.

(ii) $\operatorname{deg} f=-\infty$ if and only if $\operatorname{supp} f$ is bounded. 
(iii) If $\operatorname{deg} f \in \mathbb{Q}$, then there exist $C, R>0$ such that

$$
|f(x)| \leqslant C|x|^{\operatorname{deg} f} \quad \text { for } x \in X, \quad|x| \geqslant R .
$$

(iv) Let $\beta(f)=\min \{n \in \mathbb{Z}: n>0, n \geqslant \operatorname{deg} f\}$. Then there exist $R>0$ and $\alpha<0$ such that

$$
|f(x)| \leqslant\left(1+|x|^{2}\right)^{\beta(f)}|x|^{\alpha} \quad \text { for } x \in X, \quad|x|>R .
$$

Proof. If $\operatorname{supp} f$ is bounded, then the assertion is obvious. Assume that $\operatorname{supp} f$ is unbounded. Then the set

$$
Y=\left\{(y, f(y)) \in X \times N: \forall_{x \in X}|x|=|y| \Rightarrow 2|f(y)| \geqslant|f(x)|\right\}
$$

is unbounded and semi-algebraic. So, by the Curve Selection Lemma at infinity, there exists a curve $\psi=(\varphi, \eta):[r,+\infty) \rightarrow Y$ meromorphic at $+\infty$ such that $\eta=f \circ \varphi, \operatorname{deg} \eta \in \mathbb{Z}$, and $\operatorname{deg} \varphi>0$. Let $\theta=\operatorname{deg} \eta / \operatorname{deg} \varphi$. Then $\theta \in \mathbb{Q}$ and for some $C, D, R>0$,

$$
C|\varphi(t)|^{\theta} \leqslant|f(\varphi(t))| \leqslant D|\varphi(t)|^{\theta}, \quad t>R .
$$

The definition of $Y$ now implies that for $x \in X,|x|=|\varphi(t)|, t>R$,

$$
|f(x)| \leqslant|f(\varphi(t))| \leqslant D|\varphi(t)|^{\theta}=D|x|^{\theta} .
$$

So, $\operatorname{deg} f \leqslant \theta$. Since, by (2.2), $\operatorname{deg} f \geqslant \theta$, it follows that $\operatorname{deg} f=\theta$. This gives (i), (ii) and (iii). Part (iv) follows immediately from (iii).

2.2. $\mathscr{C}^{1}$ semi-algebraic functions. Let $f: \mathbb{R}^{n} \rightarrow \mathbb{R}$ be a semi-algebraic function of class $\mathscr{C}^{1}$ in $x=\left(x_{1}, \ldots, x_{n}\right)$. Then the gradient $\nabla f=\left(\frac{\partial f}{\partial x_{1}}, \ldots, \frac{\partial f}{\partial x_{n}}\right): \mathbb{R}^{n} \rightarrow \mathbb{R}^{n}$ is a semi-algebraic mapping.

Proposition 2.4. There exist $C, \delta, R>0$ such that

$$
\begin{gathered}
|f(x)| \geqslant R \Rightarrow|x||\nabla f(x)| \geqslant C|f(x)|, \\
|f(x)| \leqslant \delta \Rightarrow|x||\nabla f(x)| \geqslant C|f(x)| .
\end{gathered}
$$

In particular, the set $K_{\infty}(f)$ is finite. The assertion also holds for complex polynomials.

Proof. As in [19] and [6], we use Hörmander's method. To prove (2.3), assume the contrary. Then the semi-algebraic set

$$
X=\left\{(x, y, z, \varepsilon) \in \mathbb{R}^{2 n} \times \mathbb{R}^{2}: y=\nabla f(x), z=f(x),|z| \geqslant \varepsilon, \varepsilon|y||x|<|z|\right\}
$$

has an accumulation point of the form $\left(x_{0}, y_{0}, z_{0},+\infty\right)$. Thus, by the Curve Selection Lemma at infinity there exists a curve $\psi=\left(\varphi, \tau, \eta_{1}, \eta_{2}\right):[r,+\infty) \rightarrow X$ meromorphic at infinity such that $\psi(t) \rightarrow\left(x_{0}, y_{0}, z_{0},+\infty\right)$ as $t \rightarrow+\infty$. Then $\operatorname{deg} \eta_{2}>0, \operatorname{deg} \eta_{1}>0, \operatorname{deg} \varphi>0$, and

$$
\operatorname{deg} \eta_{2}+\operatorname{deg} \tau+\operatorname{deg} \varphi \leqslant \operatorname{deg} \eta_{1} .
$$

On the other hand,

$$
\operatorname{deg} \eta_{1}=\operatorname{deg} \eta_{1}^{\prime}+1=\operatorname{deg}(f \circ \varphi)^{\prime}+1 \leqslant \operatorname{deg} \tau+\operatorname{deg} \varphi,
$$

and we obtain a contradiction. Analogously we prove (2.4) and the assertion in the complex case. 
2.3. Łojasiewicz exponent. For three semi-algebraic sets $X, Y, Z \subset M$ such that $X \cap Y \subset Z$, we define a regular separation exponent of $Y$ and $Z$ on $X$ at a point $x_{0} \in X \cap Y$ to be any real positive $\theta$ such that

$$
\varrho(x, Y) \geqslant C \varrho(x, Z)^{\theta} \quad \text { for } x \in X \cap \Omega,
$$

where $C>0$ and $\Omega$ is a neighbourhood of $x_{0}$. The infimum of all such exponents $\theta$ will be denoted by $\mathcal{L}_{x_{0}}(X ; Y, Z)$. By using the method of Lipschitz stratifications ([13], [14]), the following is proved in Theorem 1.5 of [20]:

Proposition 2.5. Let $X, Y, Z \subset M$ be closed semi-algebraic sets such that $X \cap Y \subset$ $Z$, and let $x_{0} \in X \cap Y$.

(i) Then $\mathcal{L}_{x_{0}}(X ; Y, Z) \in \mathbb{Q}$, and (\#) holds for $\theta=\mathcal{L}_{x_{0}}(X ; Y, Z)$, some $C>0$ and a neighbourhood $\Omega$ of $x_{0}$, provided $0^{0}=0$.

(ii) If $x_{0} \in \overline{X \backslash Z}$, then $\mathcal{L}_{x_{0}}(X ; Y, Z)$ is attained on an analytic curve, i.e. for any neighbourhood $\tilde{\Omega}$ of $x_{0}$ there exists an analytic curve $\varphi:[0, r) \rightarrow X \cap \tilde{\Omega}$ such that $\varphi((0, r)) \subset X \backslash Z$ and $\varphi(0) \in X \cap Y$, and for some constant $C_{1}>0$,

$$
C \varrho(\varphi(t), Z)^{\mathcal{L}_{x_{0}}(X ; Y, Z)} \leqslant \varrho(\varphi(t), Y) \leqslant C_{1} \varrho(\varphi(t), Z)^{\mathcal{L}_{x_{0}}(X ; Y, Z)}, t \in[0, r) .
$$

If $Z=X \cap Y$ and $x_{0} \in \overline{X \backslash Y}$, then obviously $\mathcal{L}_{x_{0}}(X ; Y, Z)$ is equal to the Eojasiewicz exponent $\mathcal{L}_{x_{0}}(X, Y)$ of $X$ and $Y$ at $x_{0}$, i.e. the optimum exponent $\theta$ in the following separation condition:

$$
\varrho(x, X)+\varrho(x, Y) \geqslant C \varrho(x, X \cap Y)^{\theta} \quad \text { for } x \in \Omega,
$$

considered in a neighbourhood $\Omega \subset M$ of $x_{0}$ for some constant $C>0$. Note that Proposition 2.5 also holds in the subanalytic case.

2.4. Stratification. By stratification of a subset $X \subset M$ we mean a decomposition of $X$ into a locally finite disjoint union

$$
X=\bigcup S_{\alpha}
$$

where the subsets $S_{\alpha}$ are called strata, such that each $S_{\alpha}$ is a connected embedded submanifold of $M$, and each $\left(\overline{S_{\alpha}} \backslash S_{\alpha}\right) \cap X$ is the union of some strata of dimension smaller than $\operatorname{dim} S_{\alpha}$.

The $i$-th skeleton of the stratification (2.5) is

$$
X^{i}=\bigcup_{\operatorname{dim} S_{\alpha} \leqslant i} S_{\alpha} .
$$

The stratification (2.5) is called semi-algebraic if all the skeletons $X^{i}$ are semialgebraic sets (or equivalently if the number of strata is finite and they are all semi-algebraic). The stratification (2.5) of a complex algebraic subset $X$ of a complex linear space $M$ is called complex algebraic if all the skeletons $X^{i}$ are complex algebraic subsets of $M$ and the number of strata is finite.

By Corollaries 2.6 and 2.7 in [20] we have:

Proposition 2.6. Let $X, Y, Z \subset M$ be closed semi-algebraic sets such that $X \cap Y \subset$ $Z$. Then there exists a stratification

$$
X \cap Y=S_{1} \cup \cdots \cup S_{k}
$$

of $X \cap Y$ such that the function

$$
X \cap Y \ni x \mapsto \mathcal{L}_{x}(X ; Y, Z)
$$


is constant on each stratum $S_{i}$. In particular, the function (2.7) is upper semicontinuous. If additionally $X_{1}, \ldots, X_{n} \subset X \cap Y$ are semi-algebraic sets, then one can require that the stratification (2.6) is compatible with any $X_{j}$, i.e. any $X_{j}$ is a union of some strata $S_{i}$.

\section{Proof of Theorem 1.2}

Let $X \subset M$ be a closed semi-algebraic set, and let $g: X \rightarrow N$ and $f: X \rightarrow L$ be continuous semi-algebraic mappings.

The values $\vartheta_{g / f}(\lambda) \in\{-\infty,+\infty\}$ are characterised by the following:

Remark 3.1. (i) By Proposition 2.3 and the definition of $\mathfrak{S}_{f}$ we have:

$$
\vartheta_{g / f}(\lambda)=+\infty \quad \Longleftrightarrow \quad \lambda \in L \backslash \mathfrak{S}_{f} .
$$

(ii) Let $h=\left.f\right|_{g^{-1}(0)}$. From the definition of $\mathcal{L}_{\infty, f \rightarrow \lambda}(g)$ we have:

$$
\vartheta_{g / f}(\lambda)=-\infty \Leftrightarrow \lambda \in \mathfrak{S}_{h} \Leftrightarrow \mathcal{L}_{\infty}\left(f-\lambda \mid g^{-1}(0)\right)<0 .
$$

Before the proof of Theorems 1.2 we give four lemmas and a proposition. Let $B=\{z \in M:|z|<1\}$ and let $H: B \rightarrow M$ be of the form

$$
H(z)=\frac{z}{1-|z|^{2}} .
$$

Lemma 3.2. The mapping $H$ is semi-algebraic and invertible with inverse

$$
H^{-1}(x)=\frac{2 x}{1+\sqrt{1+4|x|^{2}}}
$$

Moreover, for any $R>0$,

$$
|H(z)| \geqslant R \quad \Longleftrightarrow \quad \frac{2 R}{1+\sqrt{1+4 R^{2}}} \leqslant|z|<1 .
$$

Proof. $H$ is a semi-algebraic mapping as the restriction of a rational mapping to the semi-algebraic set $B$. By an easy calculation we obtain (3.3) and the formula for $H^{-1}$.

By Lemma 3.2 we may define the following semi-algebraic sets:

$$
\begin{aligned}
Y & =\{(x, \lambda, \delta) \in X \times L \times \mathbb{R}:|f(x)-\lambda| \leqslant \delta\}, \\
Z_{1} & =\{(z, \lambda, \delta) \in B \times L \times \mathbb{R}:(H(z), \lambda, \delta) \in Y\}, \\
Z_{2} & =\partial B \times L \times \mathbb{R}, \\
Z & =Z_{1} \cup Z_{2} .
\end{aligned}
$$

Let $V=g^{-1}(0)$, and let

$$
W=\left\{(z, \lambda, \delta) \in Z_{1}: H(z) \in V\right\} .
$$

Define a mapping $F: Z \rightarrow \mathbb{R}$ by

$$
F(z, \lambda, \delta)=\left(1-|z|^{2}\right) \varrho((z, \lambda, \delta), W)
$$

Since $W$ is a semi-algebraic set, Proposition 2.1 implies that $F$ is a semi-algebraic mapping.

For any $\lambda \in L, \delta \geqslant 0$ and $S \subset X$ we set

$$
S_{\lambda, \delta}=\{x \in S:|f(x)-\lambda| \leqslant \delta\} .
$$


Lemma 3.3. Let $\lambda_{0} \in L$ and $\delta_{0}>0$ be such that the set $V_{\lambda_{0}, \delta_{0}}$ is bounded, and suppose $X_{\lambda_{0}, \delta}$ is unbounded for any $\delta>0$. Then there exist $C, D, R>0$ such that for any $(x, \lambda, \delta) \in Y$, where $0<\delta \leqslant \frac{\delta_{0}}{2}$ and $\left|\lambda-\lambda_{0}\right| \leqslant \delta$, we have

$$
C|x|^{-1} \leqslant F\left(H^{-1}(x), \lambda, \delta\right) \leqslant D|x|^{-1}, \quad x \in X_{\lambda_{0}, \delta}, \quad|x| \geqslant R .
$$

Proof. Let $Z^{\delta}=\left\{(z, \lambda, \delta) \in Z_{1}:\left|\lambda-\lambda_{0}\right| \leqslant \delta\right\}$. Then $Z^{\delta^{\prime}} \subset Z^{\delta^{\prime \prime}}$ if $\delta^{\prime} \leqslant \delta^{\prime \prime}$. By the definition of $F$ we have

$$
F(z, \lambda, \delta)=|H(z)|^{-1}|z| \varrho((z, \lambda, \delta), W) \quad \text { for } \quad(z, \lambda, \delta) \in Z_{1}, \quad z \neq 0 .
$$

Hence, by (3.3), it suffices to prove that for some $c, d, r>0$, with $r<1$, and $\delta_{1}=\frac{\delta_{0}}{2}$,

$$
c \leqslant|z| \varrho((z, \lambda, \delta), W) \leqslant d \quad \text { for } \quad(z, \lambda, \delta) \in Z^{\delta_{1}}, \quad r \leqslant|z|<1 .
$$

Because $Z^{\delta_{1}}$ is bounded, the set $\left\{|z| \varrho((z, \lambda, \delta), W):(z, \lambda, \delta) \in Z_{\lambda_{0}, \delta_{1}}\right\}$ is also bounded. Hence the right-hand estimate in (3.5) holds. By (3.3) and the assumptions on $V_{\lambda_{0}, \delta_{0}}$ and $X_{\lambda_{0}, \delta}$, there exists $0<r<1$ for which the set $W$ has no accumulation points in $A=\left\{(z, \lambda, \delta) \in Z^{\delta_{1}}: r \leqslant|z|\right\}$. Moreover, $A$ is bounded, so $c=\inf \{|z| \varrho((z, \lambda, \delta), W):(z, \lambda, \delta) \in A\}>0$. This gives the left-hand estimate in (3.5).

Let $X_{H}=H^{-1}(X) \cup \partial B$ and $V_{H}=H^{-1}(V)$. Since $g$ and $H$ are semi-algebraic mappings the sets $V, X_{H}, V_{H}$ are semi-algebraic. Moreover, $X_{H}$ is closed and $V_{H}=(g \circ H)^{-1}(0)$. Define $g_{H}: X_{H} \rightarrow N$ by

$$
g_{H}(z)= \begin{cases}\frac{g \circ H(z)}{\left(1+|H(z)|^{2}\right)^{\beta(g)}} & \text { for } z \in X_{H} \cap B, \\ 0 & \text { for } z \in \partial B,\end{cases}
$$

where $\beta(g)$ is defined in Proposition 2.3 (iv).

Lemma 3.4. The mapping $g_{H}$ is continuous, semi-algebraic and

$$
\left(g_{H}\right)^{-1}(0)=V_{H} \cup \partial B .
$$

Proof. By (2.1) in Proposition 2.3, $g_{H}$ is continuous. Since the mapping $g$ is semialgebraic, so is $B \ni x \mapsto g \circ H(x)$, and hence also $h:\left(X_{H} \cap B\right) \ni z \mapsto(g(z),(1+$ $\left.\left.|H(z)|^{2}\right)^{\beta(g)}\right) \in N \times \mathbb{R}$. The graph of $g_{H}$ is the union of $\partial B \times\{0\}$ and the image of the graph $h$ under the semi-algebraic mapping $M \times N \times(0,+\infty) \ni(z, y, t) \mapsto\left(z, \frac{1}{t} y\right) \in$ $M \times N$, so the graph of $g_{H}$ is semi-algebraic. The equality (3.6) is obvious.

The set $Z$ is semi-algebraic and $X_{H}$ is its image under the projection map $Z \ni$ $(z, \lambda, \delta) \mapsto z \in M$. Hence, we may define a semi-algebraic mapping $G: Z \rightarrow N$ by

$$
G(z, \lambda, \delta)=g_{H}(z) \text {. }
$$

Let $\Gamma$ be the graph of the semi-algebraic mapping $(G, F): Z \rightarrow N \times \mathbb{R}$. Since $Z$ is a closed set, so is $\Gamma$.

Lemma 3.5. There exists a stratification

$$
G^{-1}(0)=S_{1} \cup \cdots \cup S_{j}
$$

such that the function

$$
\mathfrak{L}: G^{-1}(0) \ni v \mapsto \mathcal{L}_{(v, 0,0)}(\Gamma ; Z \times\{0\} \times \mathbb{R}, Z \times N \times\{0\})
$$


is constant on each stratum $S_{i}$. In particular, the set of values of $\mathfrak{L}$ is a finite subset of $\mathbb{Q}$.

Proof. By (3.6),$G^{-1}(0)=F^{-1}(0)$, so

$$
G^{-1}(0) \times\{0\} \times\{0\}=\Gamma \cap(Z \times\{0\} \times \mathbb{R}) \subset Z \times N \times\{0\} .
$$

Proposition 2.5 now shows that the values of $\mathfrak{L}$ are rational numbers. Moreover, from Proposition 2.6 we obtain a stratification (3.7) satisfying the assertion.

Take any $\lambda_{0} \in L$ and define

$$
l_{\lambda_{0}}(g)=\max \left\{\mathfrak{L}\left(z, \lambda_{0}, 0\right):\left(z, \lambda_{0}, 0\right) \in Z_{2}\right\} .
$$

By Lemma 3.5, $l_{\lambda_{0}}(g) \in \mathbb{Q}$.

Proposition 3.6. Let $\delta_{0}>0$ be such that the set $V_{\lambda_{0}, \delta_{0}}$ is bounded, and suppose the set $X_{\lambda_{0}, \delta}$ is unbounded for any $\delta>0$. Then

$$
\mathcal{L}_{\infty, f \rightarrow \lambda_{0}}(g)=2 \beta(g)-l_{\lambda_{0}}(g)
$$

and for any sufficiently small $0<\delta \leqslant \frac{\delta_{0}}{2}$ there exist $C, C^{\prime}, R>0$ such that

$$
|g(x)| \geqslant C|x|^{2 \beta(g)-l_{\lambda_{0}}(g)} \quad \text { for } x \in X_{\lambda_{0}, \delta}, \quad|x| \geqslant R
$$

and

$$
C^{\prime}|\varphi(t)|^{2 \beta(g)-l_{\lambda_{0}}(g)} \geqslant|g(\varphi(t))| \geqslant C|\varphi(t)|^{2 \beta(g)-l_{\lambda_{0}}(g)}, t \in[r,+\infty),
$$

for some curve $\varphi:[r,+\infty) \rightarrow X_{\lambda_{0}, \delta}$ meromorphic at $+\infty$, with $\operatorname{deg} \varphi>0$.

Proof. Let $E=\left\{(z, \lambda, \delta) \in Z_{2}: \lambda=\lambda_{0}, \delta=0\right\}$ and $\alpha=l_{\lambda_{0}}(g)$. By the definition of $l_{\lambda_{0}}(g)$, for any $\left(z, \lambda_{0}, 0\right) \in E$ there exist a neighbourhood $\Omega_{z} \subset M \times L \times \mathbb{R}$ of $\left(z, \lambda_{0}, 0\right)$ and $C_{z}>0$ such that

$$
|G(y, \lambda, \delta)| \geqslant C_{z}|F(y, \lambda, \delta)|^{\alpha}, \quad(y, \lambda, \delta) \in \Omega_{z} \cap Z .
$$

Since the set $E$ is compact, there exists $\tilde{C}>0$ such that $C_{z} \geqslant \tilde{C}$ for $\left(z, \lambda_{0}, 0\right) \in E$, and there exist $0<r_{1}<1$ and $0<\delta_{1} \leqslant \frac{\delta_{0}}{2}$ such that

$$
\left|G\left(y, \lambda, \delta_{1}\right)\right| \geqslant \tilde{C}\left|F\left(y, \lambda, \delta_{1}\right)\right|^{\alpha}, \quad\left|\lambda-\lambda_{0}\right| \leqslant \delta_{1}, \quad r_{1} \leqslant|y|<1,
$$

where $\left(y, \lambda, \delta_{1}\right) \in Z$. Consequently,

$$
\frac{|g(x)|}{\left(1+|x|^{2}\right)^{\beta(g)}} \geqslant \tilde{C}\left|F\left(H^{-1}(x), \lambda_{0}, \delta_{1}\right)\right|^{\alpha}, \quad x \in X_{\lambda_{0}, \delta_{1}}, \quad|x| \geqslant R,
$$

where $R>0$ is the unique solution of the equation $r_{1}=\frac{2 R}{1+\sqrt{1+4 R^{2}}}$. Together with (3.4) this gives

$$
|g(x)| \geqslant \tilde{C} C\left(1+|x|^{2}\right)^{\beta(g)}|x|^{-\alpha} \quad \text { for } x \in X_{\lambda_{0}, \delta_{1}}, \quad|x| \geqslant R .
$$

Hence for any $0<\delta \leqslant \delta_{1}$, (3.10) follows.

Take any $0<\delta \leqslant \delta_{1}$. Let $\left(z_{0}, \lambda_{0}, 0\right) \in Z_{2}$ be a point such that $\mathfrak{L}\left(z_{0}, \lambda_{0}, 0\right)=$ $l_{\lambda_{0}}(g)$. By the assumption on $V_{\lambda_{0}, \delta_{0}}$ we have

$$
\left(z_{0}, \lambda_{0}, 0,0,0\right) \in \overline{\Gamma \backslash(Z \times N \times\{0\})}, \quad\left(z_{0}, \lambda_{0}, 0\right) \notin \bar{W},
$$

and $\mathfrak{L}\left(z_{0}, \lambda_{0}, 0\right)>0$. Thus, by Proposition 2.5, for any sufficiently small neighbourhood $\tilde{\Omega}$ of $\omega=\left(z_{0}, \lambda_{0}, 0, G\left(z_{0}, \lambda_{0}, 0\right), F\left(z_{0}, \lambda_{0}, 0\right)\right)=\left(z_{0}, \lambda_{0}, 0,0,0\right)$ there exists an analytic curve

$$
\psi=\left(\psi_{1}, \psi_{2}, \psi_{3}\right):[0, r) \rightarrow \Gamma \cap \tilde{\Omega},
$$


where $\psi_{1}:[0, r) \rightarrow Z, \psi_{2}=G \circ \psi_{1}:[0, r) \rightarrow N, \psi_{3}=F \circ \psi_{1}:[0, r) \rightarrow \mathbb{R}$, $\psi((0, r)) \subset \Gamma \backslash(Z \times N \times\{0\})$ and $\psi(0) \in \Gamma \cap(Z \times\{0\} \times \mathbb{R})$, such that for some constant $C_{1}>0$,

$$
\varrho(\psi(t), Z \times\{0\} \times \mathbb{R}) \leqslant C_{1} \varrho(\psi(t), Z \times M \times\{0\})^{\alpha} \quad \text { for } t \in[0, r) .
$$

Let $\varphi_{1}:[0, r) \rightarrow M, \varphi_{2}:[0, r) \rightarrow L, \varphi_{3}:[0, r) \rightarrow \mathbb{R}$, and let $\psi_{1}=\left(\varphi_{1}, \varphi_{2}, \varphi_{3}\right)$. By the choice of $\psi$ we have $\varphi_{1}(t) \in B$ for $t \in(0, r)$, and $\varphi(0) \in \partial B$ by (3.12). Hence,

$$
\left|H\left(\varphi_{1}(t)\right)\right| \rightarrow \infty \quad \text { as } t \rightarrow 0 .
$$

Since the neighbourhood $\tilde{\Omega}$ of $\omega$ can be small, one can assume that $0 \leqslant \varphi_{3}(t)<\delta$ for $t \in[0, r)$. Then, by the definition of $Z$, we have $\left|H\left(\varphi_{1}(t)\right)-\lambda_{0}\right| \leqslant \varphi_{3}(t)<\delta$ for $t \in(0, r)$, and so

$$
H\left(\varphi_{1}(t)\right) \in X_{\lambda_{0}, \delta} \quad \text { for } t \in(0, r) .
$$

By (3.13),

$$
\left|G\left(\psi_{1}(t)\right)\right| \leqslant C_{1}\left|F\left(\psi_{1}(t)\right)\right|^{\alpha} \quad \text { for } t \in[0, r] .
$$

Hence, from (3.4) and (3.14), for some $0<r_{1}<r$,

$$
\frac{\left|g\left(H\left(\varphi_{1}(t)\right)\right)\right|}{\left(1+\left|H\left(\varphi_{1}(t)\right)\right|^{2}\right)^{\beta(g)}} \leqslant C_{1} D^{l_{\lambda_{0}}(g)}\left|H\left(\varphi_{1}(t)\right)\right|^{-\alpha}, \quad t \in\left(0, r_{1}\right] .
$$

Together with (3.14) and (3.15), this gives

$$
\left|g\left(H\left(\varphi_{1}(t)\right)\right)\right| \leqslant C^{\prime}\left|H\left(\varphi_{1}(t)\right)\right|^{2 \beta(g)-\alpha}, \quad t \in\left(0, r_{1}\right]
$$

for some $C^{\prime}>0$. Now setting $\varphi(t)=H\left(\varphi_{1}\left(\frac{1}{t}\right)\right)$ for $t \in\left[\frac{1}{r_{1}},+\infty\right)$ we obtain (3.11). Finally, (3.11) and (3.10) yield (3.9).

Proof of Theorem 1.2. Fix $\lambda_{0} \in L$. First we prove (i). If for any $\delta>0$ the set $V_{\lambda_{0}, \delta}$ is unbounded, then $\mathcal{L}_{\infty, f \rightarrow \lambda_{0}}(g)=-\infty$. If for some $\delta>0$ the set $X_{\lambda_{0}, \delta}$ is bounded, then $\mathcal{L}_{\infty, f \rightarrow \lambda_{0}}(g)=+\infty$. The remaining case in (i) follows from the fact that $\beta(g) \in \mathbb{Z}$ (see Proposition 2.3) and from (3.9) in Proposition 3.6.

To prove (ii), we adopt the method of the proof of Theorem 3.2.2 in [18. By Lemma 3.5, let

$$
\vartheta_{g / f}(L)=\left\{r_{1}, \ldots, r_{s}\right\} \subset \mathbb{Q} \cup\{-\infty,+\infty\}, \text { where } r_{1} \leqslant \cdots \leqslant r_{s} .
$$

Define $\Lambda_{\xi}=\left\{\lambda \in L: \mathcal{L}_{\infty, f \rightarrow \lambda}(g) \leqslant \xi\right\}$ for $\xi \in \overline{\mathbb{R}}$.

Fix $r_{i}$. We now prove that the set $\Lambda_{r_{i}}$ is closed and semi-algebraic. If $r_{i} \in$ $\{-\infty,+\infty\}$ this follows from Remark 3.1 and Proposition 2.2. So, let $r_{i}=\frac{a}{b}$, where $a, b \in \mathbb{Z}$ and $b>0$. Define

$$
T=\left\{(x, c) \in X \times \mathbb{R}:|g(x)|^{b}=c|x|^{a}\right\},
$$

and let $p: T \ni(x, c) \mapsto(f(x), c) \in L \times \mathbb{R}$. Since the mapping $p$ is semi-algebraic, Proposition 2.2 shows that the set $\mathfrak{S}_{p}$ is also semi-algebraic.

Let $\pi: L \times \mathbb{R} \ni(y, c) \mapsto y \in L$ and observe that

$$
\Lambda_{r_{i}}=\overline{\pi\left(\mathfrak{S}_{p}\right)} \text {. }
$$

Indeed, let $\lambda \in \Lambda_{r_{i}}$, and let $U \subset L$ be a neighbourhood of $\lambda$. Take a neighbourhood $U_{1} \subset L$ of $\lambda$ such that $\overline{U_{1}} \subset U$. Then, by Proposition 3.6, there exist $C^{\prime}>0$ such that the set

$$
\left\{(x, y) \in f^{-1}\left(U_{1}\right) \times N: y=g(x),|y|^{b} \leqslant C^{\prime}|x|^{a}\right\}
$$


is unbounded. Since it is semi-algebraic, there exists a curve $\psi=(\varphi, \eta):[r,+\infty) \rightarrow$ $f^{-1}\left(U_{1}\right) \times N$ meromorphic at infinity such that $\operatorname{deg} \varphi>0, \eta=g \circ \varphi$ and

$$
\mid g\left(\left.\varphi(t)\right|^{b} \leqslant C^{\prime}|\varphi(t)|^{a}, \quad t \in[r,+\infty) .\right.
$$

Then, for some $\lambda^{\prime} \in \overline{U_{1}} \subset U$ and $0 \leqslant c \leqslant C^{\prime}$,

$$
f \circ \varphi(t) \rightarrow \lambda^{\prime} \quad \text { and } \quad \frac{|g(\varphi(t))|^{b}}{|\varphi(t)|^{a}} \rightarrow c \quad \text { as } \quad t \rightarrow \infty .
$$

Hence, $\lambda^{\prime} \in \pi\left(\mathfrak{S}_{p}\right) \cap U$, and so $\lambda \in \overline{\pi\left(\mathfrak{S}_{p}\right)}$.

Now let $\lambda \in \overline{\pi\left(\mathfrak{S}_{p}\right)}$. Take any neighbourhood $U \subset L$ of $\lambda$, and let $\lambda^{\prime} \in U$ and $c \in \mathbb{R}$ be such that $\left(\lambda^{\prime}, c\right) \in \mathfrak{S}_{p}$. Then for some sequence $\left(x_{n}, c_{n}\right) \in T$, where $x_{n} \in f^{-1}(U)$ and $c_{n} \in \mathbb{R}$ for $n \in \mathbb{N}$, we have

$$
\left|x_{n}\right| \rightarrow \infty, \quad f\left(x_{n}\right) \rightarrow \lambda^{\prime} \quad \text { and } \quad c_{n} \rightarrow c \quad \text { as } n \rightarrow \infty .
$$

Hence, there exists $C>0$ such that $\left|c_{n}\right| \leqslant C$ for $n \in \mathbb{N}$, and so

$$
\left|g\left(x_{n}\right)\right|^{b} \leqslant C\left|x_{n}\right|^{a}, \quad n \in \mathbb{N} .
$$

This gives $\mathcal{L}_{\infty}\left(g \mid f^{-1}(U)\right) \leqslant r_{i}$, and hence $\mathcal{L}_{\infty, f \rightarrow \lambda}(g) \leqslant r_{i}$. Summing up, $\lambda \in \Lambda_{r_{i}}$ and (3.16) is proved.

By Proposition 2.2 the set $\mathfrak{S}_{p}$ is semi-algebraic, so, by (3.16), $\Lambda_{r_{i}}$ is closed and semi-algebraic. In particular, the function $\vartheta_{g / f}$ is upper semi-continuous. From the definition of $\Lambda_{r_{i}}$ we have $\Lambda_{r_{1}} \mp \ldots \mp \Lambda_{r_{s}}=L$. Hence, $\Lambda_{\xi}$ is semi-algebraic for any $\xi \in \overline{\mathbb{R}}$. Therefore there exists a semi-algebraic stratification of the form (1.1) compatible with any intersection $X_{1} \cap \cdots \cap X_{j}$, where $X_{1}, \ldots, X_{j} \in\left\{\Lambda_{r_{1}}, \ldots, \Lambda_{r_{s}}\right\}$. Thus, the function $\vartheta_{g / f}$ is constant on each stratum $S_{i}$, and Theorem 1.2 is proved.

Corollary 3.7. If $\theta=\mathcal{L}_{\infty, f \rightarrow \lambda}(g) \in \mathbb{Q}$, then for some $C, C^{\prime}, R, \delta>0$,

$$
\begin{gathered}
|g(x)| \geqslant C|x|^{\theta} \quad \text { for } x \in X,|x| \geqslant R,|f(x)-\lambda|<\delta, \\
C^{\prime}|\varphi(t)|^{\theta} \geqslant|g(\varphi(t))| \geqslant C|\varphi(t)|^{\theta} \quad \text { for } t \in[r,+\infty),
\end{gathered}
$$

where $\varphi:[r,+\infty) \rightarrow X$ is a curve meromorphic at infinity such that $\operatorname{deg} \varphi>0$ and $|f(\varphi(t))-\lambda|<\delta$ for $t \in[r,+\infty)$.

Proof. The assertion follows immediately from (3.10), (3.11) and Theorem 1.2 ,

\section{Proof of Corollary 1.6}

Let $\left(z_{1}, \ldots, z_{n}\right),\left(y_{1}, \ldots, y_{m}\right)$ be the coordinates of $z \in \mathbb{C}^{n}, y \in \mathbb{C}^{m}$, respectively.

As in the proof of Theorem 1.2 we now show that for any $\xi \in \mathbb{Q} \cup\{-\infty,+\infty\}$, the set $\Lambda_{\xi}=\left\{\lambda \in \mathbb{C}^{k}: \mathcal{L}_{\infty, f \rightarrow \lambda}(g) \leqslant \xi\right\}$ is complex algebraic. For $\xi \in\{-\infty,+\infty\}$, this is obvious. Fix $\xi=\frac{a}{b}$, where $a, b \in \mathbb{Z}, b>0,(a, b)=1$.

Let $g=\left(g_{1}, \ldots, g_{m}\right)$. For any $i=1 \ldots, n$ we define algebraic sets

$$
T_{\xi}^{i}=\left\{(z, y, u) \in X \times \mathbb{C}^{m} \times \mathbb{C}: z_{i} u=1, g_{j}^{b}(z)=y_{j} z_{i}^{a}, j=1, \ldots, m\right\}
$$

if $\xi \geqslant 0$,

$$
T_{\xi}^{i}=\left\{(z, y, u) \in X \times \mathbb{C}^{m} \times \mathbb{C}: z_{i} u=1, g_{j}^{b}(z) z_{i}^{-a}=y_{j}, j=1, \ldots, m\right\}
$$

if $\xi<0$, and mappings

$$
p_{i}: T_{\xi}^{i} \ni(z, y, u) \mapsto(f(z), y, u) \in \mathbb{C}^{k} \times \mathbb{C}^{m} \times \mathbb{C} .
$$


Denote by $\mathfrak{S}_{i}$ the set of points at which $p_{i}$ is not proper, and

$$
A_{i}=\mathfrak{S}_{i} \cap\left\{(\lambda, y, u) \in \mathbb{C}^{k} \times \mathbb{C}^{m} \times \mathbb{C}: u=0\right\}, \quad i=1, \ldots, n .
$$

Since each $\mathfrak{S}_{i}$ is algebraic, so is $A_{i}$.

Let $\pi: \mathbb{C}^{k} \times \mathbb{C}^{m} \times \mathbb{C} \ni(\lambda, y, u) \mapsto \lambda \in \mathbb{C}^{k}$ and observe that

$$
\Lambda_{\xi}=\bigcup_{i=1}^{n} \overline{\pi\left(A_{i}\right)} .
$$

Indeed, let $\lambda \in \mathbb{C}^{k}$ satisfy $\mathcal{L}_{\infty, f \rightarrow \lambda}(g) \leqslant \xi$. Take any neighbourhoods $U, W \subset$ $\mathbb{C}^{k}$ of $\lambda$ such that $\bar{W} \subset U$. By Corollary 3.7, there exist $C>0$ and a curve $\varphi=\left(\varphi_{1}, \ldots, \varphi_{n}\right):[r,+\infty) \rightarrow f^{-1}(W)$ meromorphic at infinity with $\operatorname{deg} \varphi>0$ such that

$$
|g(\varphi(t))| \leqslant C|\varphi(t)|^{\xi}, \quad t \in[r, \infty) .
$$

Let $\operatorname{deg} \varphi_{i}=\operatorname{deg} \varphi$. Then $\operatorname{deg} \varphi_{i}>0$. By the definition of $\varphi$, there exists $\lambda^{\prime} \in \bar{W}$ such that

$$
f(\varphi(t)) \rightarrow \lambda^{\prime} \quad \text { as } \quad t \rightarrow \infty .
$$

By (4.2), there exists $y \in \mathbb{C}^{m}$ such that

$$
\eta(t):=\left(\frac{g_{1}^{b}(\varphi(t))}{\varphi_{i}^{a}(t)}, \ldots, \frac{g_{m}^{b}(\varphi(t))}{\varphi_{i}^{a}(t)}\right) \rightarrow y \quad \text { as } \quad t \rightarrow \infty .
$$

Since $\operatorname{deg} \varphi_{i}>0$, we may assume that $\varphi_{i}(t) \neq 0$ for $t \in[r,+\infty)$. Putting $u(t)=$ $\frac{1}{\varphi_{i}(t)}$ for $t \in[r,+\infty)$, we easily see that

$$
p_{i}(\varphi(t), \eta(t), u(t)) \rightarrow\left(\lambda^{\prime}, y, 0\right) \quad \text { as } \quad t \rightarrow \infty .
$$

Hence $\left(\lambda^{\prime}, y, 0\right) \in \mathfrak{S}_{i}$, so $\lambda^{\prime} \in U \cap \pi\left(A_{i}\right)$, and thus $\lambda \in \overline{\pi\left(A_{i}\right)}$. This gives the inclusion " $C$ " in (4.1).

We now prove " $\supset$ ". Let $\lambda \in \overline{\pi\left(A_{i}\right)}$. Take any neighbourhood $U$ of $\lambda$. Then there exists $\lambda^{\prime} \in U \cap \pi\left(A_{i}\right)$, and so $\left(\lambda^{\prime}, y, 0\right) \in \mathfrak{S}_{i}$ for some $y=\left(y_{1}, \ldots, y_{m}\right) \in \mathbb{C}^{m}$. The definitions of $A_{i}$ and $T_{\xi}^{i}$ now yield a sequence $x_{l}=\left(x_{1, l}, \ldots, x_{n, l}\right) \in f^{-1}(U), l \in \mathbb{N}$, such that $f\left(x_{l}\right) \rightarrow \lambda^{\prime}$ and

$$
\left|x_{i, l}\right| \rightarrow \infty, \quad \frac{g_{j}^{b}\left(x_{l}\right)}{x_{i, l}^{a}} \rightarrow y_{j} \quad \text { as } \quad l \rightarrow \infty, \quad j=1, \ldots, m .
$$

Consequently, there exists $C>|y|$ such that

$$
\left|g\left(x_{l}\right)\right| \leqslant C\left|x_{l}\right|^{\xi} \quad \text { for } l \in \mathbb{N} .
$$

Hence, $\mathcal{L}_{\infty}\left(g \mid f^{-1}(U)\right) \leqslant \xi$. This gives $\mathcal{L}_{\infty, f \rightarrow \lambda}(g) \leqslant \xi$, and the inclusion " $\supset$ " in (4.1) is proved.

By Theorem 1.2, the set $\vartheta_{g / f}\left(\mathbb{C}^{k}\right) \subset \mathbb{Q} \cup\{-\infty,+\infty\}$ is finite, say $\left\{r_{1}, \ldots, r_{s}\right\}$ with $r_{1}<\cdots<r_{s}$. By (4.1), the sets $\Lambda_{r_{i}}, i=1, \ldots, s$, are algebraic, and $\Lambda_{r_{1}} \varsubsetneqq$ $\cdots \varsubsetneqq \Lambda_{r_{s}}=\mathbb{C}^{k}$. Then the function $\vartheta_{g / f}$ is upper semi-continuous. Hence the usual complex stratification of $\mathbb{C}^{n}$ compatible with complex constructible sets $\Lambda_{r_{i}} \backslash \Lambda_{r_{i-1}}$ is a desired stratification. This ends the proof. 


\section{REFERENCES}

[1] R. Benedetti, J.-J. Risler, Real algebraic and semi-algebraic sets, Actualités Mathématiques, Hermann, Paris, 1990. MR1070358 (91j:14045)

[2] J. Bochnak, M. Coste, M.-F. Roy, Real algebraic geometry, Ergeb. Math. Grenzgeb., vol. 36, Springer-Verlag, Berlin, 1998. MR 1659509 (2000a:14067)

[3] J. Chądzyński, T. Krasiński, The gradient of a polynomial at infinity, Kodai Math. J. 26 (2003), 317-339. MR2018725 (2004j:32026)

[4] T. Gaffney, Fibers of polynomial mappings at infinity and a generalized Malgrange condition. Compositio Math. 119 (1999), no. 2, 157-167. MR1723126 (2001b:32054)

[5] J. Gwoździewicz, A. Płoski, Eojasiewicz exponents and singularities at infinity of polynomials in two complex variables. Colloq. Math. 103 (2005), no. 1, 47-60. MR2148949 (2006b:32038)

[6] J. Gwoździewicz, S. Spodzieja, The Lojasiewicz gradient inequality in a neighbourhood of the fibre, Ann. Polon. Math. 87 (2005), no. 1, 151-163. MR2208542 (2007b:14137)

[7] H. V. Ha, Nombres de Eojasiewicz et singularités à l'infini des polynômes de deux variables complexes, C. R. Acad. Sci. Paris 311 (1990), 429-432. MR1075664 (91i:32033)

[8] Z. Jelonek, The set of points at which a polynomial map is not proper, Ann. Polon. Math. 58 (1993), no. 3, 259-266. MR1244397 (94i:14018)

[9] Z. Jelonek, On the generalized critical values of a polynomial mapping, Manuscripta Math. 110 (2003), no. 2, 145-157. MR 1962530 (2004c:58082)

[10] T. C. Kuo, Characterizations of v-sufficiency of jets, Topology 11 (1972) 115-131. MR0288775 (44:5971)

[11] K. Kurdyka, P. Orro, S. Simon, Semialgebraic Sard theorem for generalized critical values, J. Differential Geom. 56 (2000), no. 1, 67-92. MR1863021 (2003c:58008)

[12] S. Łojasiewicz, Sur le problème de la division, Studia Math. 18 (1959), 87-136; Rozprawy Matem. 22 (1961). MR0107168 (21:5893) MR0126072 (23:A3369)

[13] T. Mostowski, Lipschitz equisingularity, Dissertationes Math. 243 (1985), 46 pp. MR808226 (87e:32008)

[14] A. Parusiński, Lipschitz stratification of subanalytic sets, Ann. Sci. École Norm. Sup. 27 (1994), 661-696. MR1307677 (96g:32017)

[15] A. Parusiński, On the bifurcation set of complex polynomial with isolated singularities at infinity, Compositio Math. 97 (1995), 369-384. MR.1353280(96i:32038)

[16] F. Pham, Vanishing homologies and the n-variables saddlepoint method, Proc. Symposia Pure Math. 40, Part 2, Amer. Math. Soc., Providence, RI, 1983, 319-333. MR713258 (85d:32026)

[17] P. J. Rabier, Ehresmann fibrations and Palais-Smale conditions for morphisms of Finsler manifolds. Ann. of Math. (2) 146 (1997), no. 3, 647-691. MR1491449 (98m:58020)

[18] T. Rodak, Wyktadnik Łojasiewicza w pobliżu poziomicy. Ph.D. thesis, University of Łódź, 2005 (in Polish).

[19] S. Spodzieja, Lojasiewicz inequality at infinity for the gradient of a polynomial, Bull. Acad. Polon. Sci. Math. 50 (2002), 273-281. MR1948075 (2003k:14080)

[20] S. Spodzieja, The Eojasiewicz exponent of subanalytic sets, Ann. Polon. Math. 87 (2005), no. 1, 247-263. MR2208551 (2006k:32011)

[21] A. N. Varchenko, Theorems on the topological equisingularity of families of algebraic varieties and families of polynomial mappings, Math. USSR Izv. 6 (1972), 949-1008. MR0337956(49:2725)

[22] J. L. Verdier, Stratifications de Whitney et théorème de Bertini-Sard. Invent. Math. 36 (1976), 295-312. MR0481096 (58:1242)

[23] A. H. Wallace, Linear sections of algebraic varieties. Indiana Univ. Math. J. 20 (1970/1971), 1153-1162. MR0285534 (44:2752)

Faculty of Mathematics and Computer Science, University of Łódź, S. Banacha 22, 90-238 ŁóDŹ, POLAND

E-mail address: rodakt@math.uni.lodz.pl

Faculty of Mathematics and Computer Science, University of Łódź, S. Banacha 22, 90-238 ŁÓDŹ, POLAND

E-mail address: spodziej@math.uni.lodz.pl 